\title{
Atenção secundária em saúde bucal no Rio Grande do Sul: análise descritiva da produção especializada em municípios com Centros de Especialidades Odontológicas com base no Sistema de Informações Ambulatoriais do Sistema Único de Saúde
}

Secondary oral health care in the state of Rio Grande do Sul: descriptive analysis of the specialized production in cities with Dental Specialty Centers from the Outpatient Information System of the Unified Health System

Ana Luiza Cardoso Pires* Jéssika Leandra Assunção Lopes Gruendemann* Guilherme Scheeren Figueiredo* Marcus Cristian Muniz Conde ${ }^{* *}$ Marcos Britto Corrêa ${ }^{* * *}$ Luiz Alexandre Chisini***

\section{Resumo}

Os Centros de Especialidades Odontológicas (CEOs) são estabelecimentos que servem de referência para unidades básicas de saúde, devem estar vinculados ao Cadastro Nacional de Estabelecimentos de Saúde (CNES) e ofertar procedimentos odontológicos especializados à população. Objetivo: este estudo tem como objetivo descrever a produção odontológica especializada nos municípios do Rio Grande do Sul com CEOs, com base nos dados do Sistema de Informações Ambulatoriais do Sistema Único de Saúde (SIA SUS). Materiais e método: foi conduzido um estudo do tipo longitudinal retrospectivo com utilização de dados secundários do SIA SUS. Realizou-se uma consulta no CNES dos CEOs cadastrados em municípios gaúchos. A produção odontológica dessas cidades foi então pesquisada diretamente no banco de dados do Departamento de Informática do SUS (Data SUS) e posteriormente tabulada. A partir disso, criou-se uma série histórica para cada cidade.
A produção mínima mensal variou de acordo com as modalidades de CEOs (Portaria Interministerial no 1.464 de 2011) e o número de CEOs por município. Resultados: procedimentos da área de periodontia foram os predominantes $(46,2 \%)$, seguidos de cirurgia oral menor $(41,3 \%)$ e endodontia (12,5\%). Quanto ao cumprimento das metas, Pelotas foi o município com melhor desempenho (91\%), seguido de Caxias do Sul (90\%) e Canoas (86\%). Em contraponto, treze municípios atingiram apenas $25 \%$ ou menos das metas estabelecidas. Conclusão: há uma vasta diferença no cumprimento ou registro dos procedimentos odontológicos especializados entre os municípios com CEOs do RS.

Palavras-chave: Saúde Pública. Avaliação dos serviços de saúde. Serviços de saúde bucal. Especialidades odontológicas. Saúde bucal.

Graduandos de Odontologia, Faculdade de Odontologia, Universidade Federal de Pelotas, RS, Brasil.

*ós-doutorando em Odontologia, Universidade Federal de Pelotas, RS, Brasil.

** Professor adjunto, Universidade Federal de Pelotas, RS, Brasil.

*** Mestrando em Odontologia, Universidade Federal de Pelotas, RS, Brasil. 


\section{Introdução}

A saúde pública no Brasil consolida-se como direito universal e dever de todas as esferas de governo com a Constituição federal de $1988^{1}$. Com um conceito ampliado e vinculado às políticas sociais e econômicas, a saúde pública busca atuar de forma integral, não meramente prestando assistência, mas, sim, prevenindo e promovendo a saúde ${ }^{2}$. As últimas duas décadas foram anos de extrema importância e de mudança na saúde pública do Brasil. Em 1994, quando o Programa Saúde da Família foi apresentado, iniciou-se uma estratégia de consolidação do Sistema Único de Saúde (SUS) ${ }^{3}$, em uma tentativa de inverter o modelo assistencialista vigente.

Assim, houve uma mudança na concepção de saúde, que anteriormente era compreendida como ausência de doença ${ }^{4,5}$, excluindo os demais usuários dos focos de atenção e atuando pontualmente nos doentes. Atualmente, compreendendo-se a saúde como um processo e não uma situação estática, pode-se identificar os grupos de risco e criar estratégias preventivas em saúde. Dessa forma, modelos centrados apenas na doença e em hospitais estão sendo substituídos por modelos que priorizam a proteção e a promoção à saúde, entendendo o indivíduo como parte integrante de uma comunidade, de forma equânime, universal e contínua ${ }^{6,7}$.

O Brasil Sorridente, como parte dessas novas políticas, visou contemplar a saúde bucal com o objetivo de universalizar o seu acesso. Sua efetivação proporcionou a reorganização da atenção básica, a ampliação e a qualificação da atenção especializada por meio da implantação dos Centros de Especialidades Odontológicas (CEOs) e dos Laboratórios Regionais de Prótese Dentária ${ }^{8,9}$. Os CEOs, que são unidades de atenção secundária à saúde bucal, devem estar integrados ao planejamento locorregional e devem proporcionar assim, pelo sistema de referência e contrarreferência, a disponibilidade de procedimentos de média complexidade aos usuários do SUS ${ }^{10,11}$.

Atualmente, os Centros de Especialidades Odontológicas estão divididos em três tipos: CEOs tipo I (três cadeiras odontológicas), aos quais são destinados mensalmente $\mathrm{R} \$ 8.250,00$ para custeio; CEOs tipo II (com quatro ou mais cadeiras), cujo valor mensal recebido é de $R \$ 11.000,00$, e CEOs tipo III (possuem, no mínimo, sete cadeiras), cujo recurso de custeio é no valor de $\mathrm{R} \$ 19.250,00$. Esses centros devem funcionar 40 horas semanais, com um número de profissionais variável em função do tipo de CEO. Os pacientes atendidos nesses locais são encaminhados pelas unidades básicas de saúde ou pelas Equipes de Saúde da Família ${ }^{12}$.

Assim sendo, os CEOs devem contemplar no mínimo as áreas de diagnóstico bucal, com ênfase em diagnóstico e detecção do câncer bucal, periodontia especializada, cirurgia oral menor dos tecidos moles e duros, endodontia e procedimentos básicos aos pa- cientes com necessidades especiais. A produção mínima mensal a ser realizada nos CEOs varia de acordo com a modalidade de cada um. CEOs tipo I devem realizar 80 procedimentos básicos, 60 de periodontia, 35 de endodontia e 80 de cirurgia oral, nos CEOs tipo II, 110 procedimentos básicos, 90 de periodontia, 60 de endodontia e 90 de cirurgia oral, já nos CEOs tipo III, 190 procedimentos básicos, 150 de periodontia, 95 de endodontia e 170 de cirurgia oral menor ${ }^{13}$.

Uma das formas de monitoramento das unidades básicas de saúde e dos centros de especialidades é pelo lançamento dos dados referentes aos procedimentos realizados no sistema informatizado do Ministério da Saúde. Essas informações, por sua vez, estão disponíveis em um grande banco de dados de domínio público. Além disso, o Ministério da Saúde prevê a utilização desse banco de dados como uma das ferramentas de gestão para a avaliação dos serviços, que é de fundamental importância como instrumento de planejamento, orientação e consolidação das políticas públicas ${ }^{14}$.

Apesar de encontrarmos centros de especialidades cadastrados há mais de dez anos no RS, a literatura carece de trabalhos que descrevam seu desempenho. Sendo assim, o presente trabalho tem o objetivo de descrever o cumprimento das metas da produção odontológica especializada do Rio Grande do Sul, para assim compreender o panorama regional e contribuir como instrumento das novas políticas públicas de saúde.

\section{Materiais e método}

Foi conduzido um estudo do tipo longitudinal retrospectivo com utilização de dados secundários do Sistema de Informações Ambulatoriais do SUS. As variáveis de interesse foram:

a) os municípios com CEOs cadastrados no Cadastro Nacional de Estabelecimentos de Saúde;

b) os procedimentos odontológicos descritos na Portaria Interministerial $\mathrm{n}$ - 1.464 , de 24 de junho de 2011, que institui o financiamento dos CEOs.

Dessa forma, realizou-se uma busca pelos CEOs dos municípios gaúchos cadastrados no CNES (disponível no portal: www.cnes.datasus.gov.br). Foram incluídos na amostra todos os CEOs encontrados e que se apresentavam ativos.

A produção odontológica, referente à Portaria 1.464, de 24 de junho de 2011, nesses municípios foi então pesquisada diretamente no banco de dados do Departamento de Informática do SUS (Data SUS), no item Informações de saúde, subitem produção ambulatorial, por gestor - a partir de 2008. Os dados pesquisados foram tabulados pelo programa TabWin, do Departamento de Informática do SUS e posteriormente exportados para o programa de planilhas Microsoft Excel, versão 2010. 
Posteriormente à tabulação, criou-se uma série histórica (de janeiro de 2013 a março de 2015) para cada cidade. Esses procedimentos foram agrupados em quatro subgrupos de procedimentos: bási$\cos$, de periodontia, de endodontia e de cirurgia oral menor, de acordo com a Portaria Interministerial 1.464/2011. Para analisar o cumprimento das metas, criou-se um critério denominado indicador do cumprimento das metas. Esse indicador foi criado a partir de cada subgrupo de procedimento, que foi avaliado mês a mês, município a município, quanto ao cumprimento das metas referente ao tipo e ao número de CEOs cadastrados para cada cidade. Os dados foram tabulados dicotomicamente em uma planilha entre os que atingiam e não atingiam as metas estabelecidas. A coleta de dados foi realizada durante o mês de junho de 2015. Os dados foram analisados de maneira descritiva, estimando-se frequências absolutas e relativas das variáveis de interesse.

\section{Resultados}

Vinte e sete CEOs foram localizados e considerados elegíveis para o estudo. Desses, 21 eram CEOs tipo I, seis eram tipo II e nenhum CEO tipo III. Os CEOs estiveram distribuídos em 23 cidades gaúchas. Porto Alegre foi o município que mais apresentou CEOs, totalizando quatro, seguido de Pelotas com dois CEOs. Os demais municípios apresentaram apenas um CEO cadastrado (Tabela 1).

Tabela 1 - Descrição dos municípios do estado do Rio Grande do Sul com CEOs cadastrados no CNES, respectivos tipos de CEO e datas de cadastro no CNES

\begin{tabular}{|c|c|c|c|}
\hline Município & População* & Tipo de CEO & Data de cadastro no CNES \\
\hline Alegrete & 78.768 & I & $30 / 03 / 2010$ \\
\hline Alvorada & 205.683 & I & $16 / 06 / 2005$ \\
\hline Bagé & 121.500 & II & $14 / 07 / 2008$ \\
\hline Bento Gonçalves & 112.318 & I & $17 / 11 / 2010$ \\
\hline Cachoeirinha & 125.246 & 1 & $04 / 11 / 2006$ \\
\hline Canguçu & 55.637 & I & 06/01/2006 \\
\hline Canoas & 339.979 & II & 05/10/2007 \\
\hline Caxias do Sul & 470.223 & II & $20 / 04 / 2005$ \\
\hline Erechim & 101.752 & 1 & $19 / 02 / 2014$ \\
\hline ljuí & 82.563 & I & $03 / 10 / 2014$ \\
\hline Montenegro & 62.861 & I & $19 / 07 / 2006$ \\
\hline Novo Hamburgo & 248.251 & II & $27 / 02 / 2015$ \\
\hline Pelotas & 342.053 & I & 09/11/2011 \\
\hline Pelotas & 342.053 & I & 03/05/2014 \\
\hline Porto Alegre & 1.472 .482 & I & 14/06/2012 \\
\hline Porto Alegre & 1.472 .482 & I & 03/12/2011 \\
\hline Porto Alegre & 1.472 .482 & I & $12 / 05 / 2013$ \\
\hline Porto Alegre & 1.472 .482 & I & 03/07/2012 \\
\hline Osório & 43.586 & I & $19 / 02 / 2014$ \\
\hline Rosário do Sul & 40.798 & I & $17 / 08 / 2013$ \\
\hline Santa Maria & 274.838 & II & $17 / 03 / 2003$ \\
\hline Santiago & 50.622 & 1 & $18 / 04 / 2008$ \\
\hline São Borja & 63.089 & I & 03/10/2008 \\
\hline São Lourenço & 44.520 & I & 08/08/2014 \\
\hline São Luiz Gonzaga & 35.266 & I & 03/06/2014 \\
\hline São Sepé & 24.448 & II & 06/05/2012 \\
\hline Tenente Portela & 14.553 & l & $10 / 05 / 2012$ \\
\hline
\end{tabular}

Fonte: CNES e IBGE, 2014.*

O maior número de procedimentos concentrou-se na área de periodontia $(46,2 \%)$ seguida por cirurgia oral menor $(41,3 \%)$. A endodontia concentrou apenas $12,5 \%$ dos procedimentos registrados (Tabela 2). Mais de 90 mil procedimentos especializados foram registrados no SIA SUS durante o período, somente Porto Alegre contribuiu com mais de 32 mil $(35,8 \%)$ procedimentos. Em contrapartida, uma expressiva quantidade de municípios apresentou um registro de produção especializada extremamente pobre, chegando a ser nulo em algumas vezes (Tabela 2). 
Uma ampla diferença de registros é observada (Figura 1) entre os municípios avaliados, principalmente em relação a procedimentos endodônticos. Quinze municípios $(55,6 \%)$ dos 27 avaliados não registraram nem ao menos um procedimento de endodontia ou periodontia. Além disso, um dos municípios não registrou nenhum procedimento em nenhuma das áreas estudadas e outro município registrou apenas um único procedimento, na área de cirurgia oral menor.

Da mesma forma, observamos uma disparidade no cumprimento das metas, pois apenas seis (26\%) dos 23 municípios atingiram mais que $50 \%$ das metas durante os 28 meses analisados (Tabela 3). Apesar de algumas cidades obterem altos índices no cumprimento das metas, a maioria dos municípios apresentou um registro que não atinge 30\% das metas estipuladas. Entre os municípios com registros significativos, Pelotas foi a cidade que obteve o melhor desempenho, atingindo o cumprimento de $91 \%$ das metas, seguido de Caxias do Sul (90\%) e Canoas (86\%). Porto Alegre, apesar de ter a maior produção absoluta dentre os municípios, atingiu $66 \%$ das metas, considerando seus quatro CEOs.

Tabela 2 - Procedimentos odontológicos especializados* registrados pelos municípios do RS com CEOs no SIA SUS, distribuídos por área

\begin{tabular}{|c|c|c|c|c|c|c|c|}
\hline & \multicolumn{2}{|c|}{ Periodontia } & \multicolumn{2}{|c|}{ Endodontia } & \multicolumn{2}{|c|}{ Cirurgia oral menor } & \multirow{2}{*}{ Total } \\
\hline & Absoluto & $\%$ & Absoluto & $\%$ & Absoluto & $\%$ & \\
\hline Alegrete & 415 & $24,17 \%$ & 469 & $27,31 \%$ & 833 & $48,52 \%$ & 1717 \\
\hline Alvorada & 0 & $0 \%$ & 0 & $0 \%$ & 268 & $100 \%$ & 268 \\
\hline Bagé & 0 & $0 \%$ & 0 & $0 \%$ & 19 & $100 \%$ & 19 \\
\hline Bento Gonçalves & 87 & $13,10 \%$ & 141 & 141 & 436 & $65,67 \%$ & 664 \\
\hline Cachoeirinha & 0 & $0 \%$ & 0 & $0 \%$ & 179 & $100 \%$ & 179 \\
\hline Canguçu & 0 & $0 \%$ & 0 & $0 \%$ & 49 & $100 \%$ & 49 \\
\hline Canoas & 8272 & $42,61 \%$ & 1269 & $6,54 \%$ & 9869 & $50,85 \%$ & 19410 \\
\hline Caxias do Sul & 3138 & $31,48 \%$ & 2907 & $29,16 \%$ & 3921 & $39,36 \%$ & 9966 \\
\hline Erechim & 0 & $0 \%$ & 0 & $0 \%$ & 50 & $100 \%$ & 50 \\
\hline ljuí & 0 & $0 \%$ & 0 & $0 \%$ & 0 & $0 \%$ & 0 \\
\hline Montenegro & 0 & $0 \%$ & 0 & $0 \%$ & 17 & $100 \%$ & 17 \\
\hline Novo Hamburgo & 424 & $95,07 \%$ & 1 & $0,23 \%$ & 21 & $4,70 \%$ & 446 \\
\hline Osório & 0 & $0 \%$ & 0 & $0 \%$ & 97 & $100 \%$ & 97 \\
\hline Pelotas & 9707 & $51,32 \%$ & 1544 & $8,16 \%$ & 7664 & $40,52 \%$ & 18915 \\
\hline Porto Alegre & 17360 & $53,91 \%$ & 3872 & $12,02 \%$ & 10968 & $34,07 \%$ & 32200 \\
\hline Rosário do Sul & 0 & $0 \%$ & 0 & $0 \%$ & 80 & $100 \%$ & 80 \\
\hline Santa Maria & 0 & $0 \%$ & 0 & $0 \%$ & 927 & $100 \%$ & 927 \\
\hline Santiago & 0 & $0 \%$ & 0 & $0 \%$ & 72 & $100 \%$ & 72 \\
\hline São Borja & 2235 & $44,81 \%$ & 1055 & $21,15 \%$ & 1697 & $34,04 \%$ & 4987 \\
\hline São Lourenço do Sul & 0 & $0 \%$ & 0 & $0 \%$ & 3 & $100 \%$ & 3 \\
\hline São Luiz Gonzaga & 0 & $0 \%$ & 0 & $0 \%$ & 3 & $100 \%$ & 3 \\
\hline São Sepé & 0 & $0 \%$ & 0 & $0 \%$ & 53 & $100 \%$ & 53 \\
\hline Tenente Portela & 0 & $0 \%$ & 0 & $0 \%$ & 1 & $100 \%$ & 1 \\
\hline Absoluto (\%) & \multicolumn{2}{|c|}{$41638(46,2 \%)$} & \multicolumn{2}{|c|}{$11258(12,5 \%)$} & \multicolumn{2}{|c|}{$37141(41,3 \%)$} & 90037 (100\%) \\
\hline
\end{tabular}

${ }^{*}$ Procedimentos especializados que os municípios com CEOs devem realizar segundo a Portaria $n^{\circ}$ 1.464, de 24 de junho de 2011. Procedimentos registrados para o período de janeiro de 2013 a abril de 2015.

Fonte: SIA SUS. 
Tabela 3 - Análise descritiva das metas atingidas e não atingidas nas cidades do Rio Grande do Sul com CEOs, distribuídas por especialidade no período de janeiro de 2013 a janeiro de 2015

\begin{tabular}{|c|c|c|c|c|c|c|c|c|c|}
\hline \multirow{2}{*}{ Municípios/metas } & \multicolumn{2}{|c|}{ Básicos } & \multicolumn{2}{|c|}{ Periodontia } & \multicolumn{2}{|c|}{ Endodontia } & \multicolumn{2}{|c|}{ Cirurgia } & \multirow{2}{*}{ Total \% } \\
\hline & Meses* & $(\%)$ & Meses* & $(\%)$ & Meses* & $(\%)$ & Meses* & $(\%)$ & \\
\hline Alegrete & & & & & & & & & $30 \%$ \\
\hline Atingida & 24 & $(96 \%)$ & 2 & $(8 \%)$ & 3 & $(12 \%)$ & 1 & $(4 \%)$ & \\
\hline Não atingida & 1 & $(4 \%)$ & 23 & $(92 \%)$ & 22 & $(88 \%)$ & 24 & $(96 \%)$ & \\
\hline Alvorada & & & & & & & & & $25 \%$ \\
\hline Atingida & 25 & $(100 \%)$ & 0 & $(0 \%)$ & 0 & $(0 \%)$ & 0 & $(0 \%)$ & \\
\hline Não atingida & 0 & $(0 \%)$ & 25 & $(100 \%)$ & 25 & $(100 \%)$ & 25 & $(100 \%)$ & \\
\hline Bagé & & & & & & & & & $25 \%$ \\
\hline Atingida & 28 & $(100 \%)$ & 0 & $(0 \%)$ & 0 & $(0 \%)$ & 0 & $(0 \%)$ & \\
\hline Não atingida & 0 & $(0 \%)$ & 28 & $(100 \%)$ & 28 & $(100 \%)$ & 28 & $(100 \%)$ & \\
\hline Bento Gonçalves & & & & & & & & & $28 \%$ \\
\hline Atingida & 28 & $(100 \%)$ & 0 & $(0 \%)$ & 2 & $(7 \%)$ & 1 & $(4 \%)$ & \\
\hline Não atingida & 0 & $(0 \%)$ & 28 & $(100 \%)$ & 26 & $(93 \%)$ & 27 & $(96 \%)$ & \\
\hline Cachoeirinha & & & & & & & & & $25 \%$ \\
\hline Atingida & 28 & $(100 \%)$ & 0 & $(0 \%)$ & 0 & $(0 \%)$ & 0 & $(0 \%)$ & \\
\hline Não atingida & 0 & $(0 \%)$ & 28 & $(100 \%)$ & 28 & $(100 \%)$ & 28 & $(100 \%)$ & \\
\hline Canguçu & & & & & & & & & $25 \%$ \\
\hline Atingida & 25 & $(100 \%)$ & 0 & $(0 \%)$ & 0 & $(0 \%)$ & 0 & $(0 \%)$ & \\
\hline Não atingida & 0 & $(0 \%)$ & 25 & $(100 \%)$ & 25 & $(100 \%)$ & 25 & $(100 \%)$ & \\
\hline Canoas & & & & & & & & & $86 \%$ \\
\hline Atingida & 27 & $(96 \%)$ & 23 & $(82 \%)$ & 21 & $(75 \%)$ & 25 & $(89 \%)$ & \\
\hline Não atingida & 1 & $(4 \%)$ & 5 & $(18 \%)$ & 7 & $(25 \%)$ & 3 & $(11 \%)$ & \\
\hline Caxias do Sul & & & & & & & & & $90 \%$ \\
\hline Atingida & 28 & $(100 \%)$ & 20 & $(70 \%)$ & 27 & $(96 \%)$ & 26 & $(92 \%)$ & \\
\hline Não atingida & 0 & $(0 \%)$ & 8 & $(29 \%)$ & 1 & $(4 \%)$ & 2 & $(8 \%)$ & \\
\hline Erechim & & & & & & & & & $25 \%$ \\
\hline Atingida & 14 & $(100 \%)$ & 0 & $(0 \%)$ & 0 & $(0 \%)$ & $(0 \%)$ & $(100 \%)$ & \\
\hline Não atingida & 0 & $(0 \%)$ & 14 & $(100 \%)$ & 14 & $(100 \%)$ & 14 & $(100 \%)$ & \\
\hline ljuí & & & & & & & & & $25 \%$ \\
\hline Atingida & 6 & $(100 \%)$ & 0 & $(0 \%)$ & 0 & $(0 \%)$ & 0 & $(0 \%)$ & \\
\hline Não atingida & 0 & $(0 \%)$ & 6 & $(100 \%)$ & 6 & $(100 \%)$ & 6 & $(100 \%)$ & \\
\hline Montenegro & & & & & & & & & $25 \%$ \\
\hline Atingida & 22 & $(100 \%)$ & 0 & $(0 \%)$ & 0 & $(0 \%)$ & 0 & $(0 \%)$ & \\
\hline Não atingida & 0 & $(30 \%)$ & 22 & $(100 \%)$ & 22 & $(100 \%)$ & 22 & $(100 \%)$ & \\
\hline Novo Hamburgo & & & & & & & & & $50 \%$ \\
\hline Atingida & 1 & $(100 \%)$ & 1 & $(100 \%)$ & 0 & $(0 \%)$ & 0 & $(0 \%)$ & \\
\hline Não atingida & 0 & $(0 \%)$ & 0 & $(0 \%)$ & 1 & $(100 \%)$ & 1 & $(100 \%)$ & \\
\hline Osório & & & & & & & & & $25 \%$ \\
\hline Atingida & 14 & $(100 \%)$ & 0 & $(0 \%)$ & 0 & $(0 \%)$ & 0 & $(0 \%)$ & \\
\hline Não atingida & 0 & $(0 \%)$ & 14 & $(100 \%)$ & 14 & $(100 \%)$ & 14 & $(100 \%)$ & \\
\hline Pelotas & & & & & & & & & $91 \%$ \\
\hline Atingida & 28 & $(100 \%)$ & 28 & $(100 \%)$ & 18 & $(64 \%)$ & 28 & $(100 \%)$ & \\
\hline Não atingida & 0 & $(0 \%)$ & 0 & $(0 \%)$ & 10 & $(36 \%)$ & 0 & $(0 \%)$ & \\
\hline Porto Alegre & & & & & & & & & $66 \%$ \\
\hline Atingida & 28 & $(100 \%)$ & 28 & $(100 \%)$ & 12 & $(42 \%)$ & 26 & $(93 \%)$ & \\
\hline Não atingida & 0 & $(0 \%)$ & 0 & $(0 \%)$ & 16 & $(58 \%)$ & 2 & $(7 \%)$ & \\
\hline
\end{tabular}


cont.

\begin{tabular}{|c|c|c|c|c|c|c|c|c|c|}
\hline Rosário do Sul & & & & & & & & & $25 \%$ \\
\hline Atingida & 20 & $(100 \%)$ & 0 & $(0 \%)$ & 0 & $(0 \%)$ & 0 & $(0 \%)$ & \\
\hline Não atingida & 0 & $(0 \%)$ & 20 & $(100 \%)$ & 20 & $(100 \%)$ & 20 & $(100 \%)$ & \\
\hline Santa Maria & & & & & & & & & $28 \%$ \\
\hline Atingida & 28 & $(100 \%)$ & 0 & $(0 \%)$ & 0 & $(0 \%)$ & 3 & $(11 \%)$ & \\
\hline Não atingida & 0 & $(0 \%)$ & 28 & $(100 \%)$ & 28 & $(100 \%)$ & 25 & $(89 \%)$ & \\
\hline Santiago & & & & & & & & & $25 \%$ \\
\hline Atingida & 28 & $(100 \%)$ & 0 & $(0 \%)$ & 0 & $(0 \%)$ & 0 & $(0 \%)$ & \\
\hline Não atingida & 0 & $(0 \%)$ & 28 & $(100 \%)$ & 28 & $(100 \%)$ & 28 & $(100 \%)$ & \\
\hline São Borja & & & & & & & & & $77 \%$ \\
\hline Atingida & 28 & $(100 \%)$ & 20 & $(71 \%)$ & 24 & $(85 \%)$ & 14 & $(50 \%)$ & \\
\hline Não atingida & 0 & $(0 \%)$ & 8 & $(29 \%)$ & 4 & $(15 \%)$ & 14 & $(50 \%)$ & \\
\hline São Lourenço do Sul & & & & & & & & & $25 \%$ \\
\hline Atingida & 7 & $(100 \%)$ & 0 & $(0 \%)$ & 0 & $(0 \%)$ & 0 & $(0 \%)$ & \\
\hline Não atingida & 0 & $(0 \%)$ & 7 & $(100 \%)$ & 7 & $(100 \%)$ & 7 & $(100 \%)$ & \\
\hline São Luiz Gonzaga & & & & & & & & & $25 \%$ \\
\hline Atingida & 10 & $(100 \%)$ & 0 & $(0 \%)$ & 0 & $(0 \%)$ & 0 & $(0 \%)$ & \\
\hline Não atingida & 0 & $(0 \%)$ & 10 & $(100 \%)$ & 10 & $(100 \%)$ & 10 & $(100 \%)$ & \\
\hline São Sepé & & & & & & & & & $25 \%$ \\
\hline Atingida & 28 & $(100 \%)$ & 0 & $(0 \%)$ & 0 & $(0 \%)$ & 0 & $(0 \%)$ & \\
\hline Não atingida & 0 & $(0 \%)$ & 28 & $(100 \%)$ & 28 & $(0 \%)$ & 28 & $(0 \%)$ & \\
\hline Tenente Portela & & & & & & & & & $11 \%$ \\
\hline Atingida & 11 & $(46 \%)$ & 0 & $(0 \%)$ & 0 & $(0 \%)$ & 0 & $(0 \%)$ & \\
\hline Não atingida & 13 & $(54 \%)$ & 24 & $(100 \%)$ & 24 & $(100 \%)$ & 24 & $(100 \%)$ & \\
\hline
\end{tabular}

* Diferença no número de meses avaliados deve-se ao fato de os municípios apresentarem CEOs cadastrados em dlferente períodos de tempo.

Fonte: SIA/SUS.

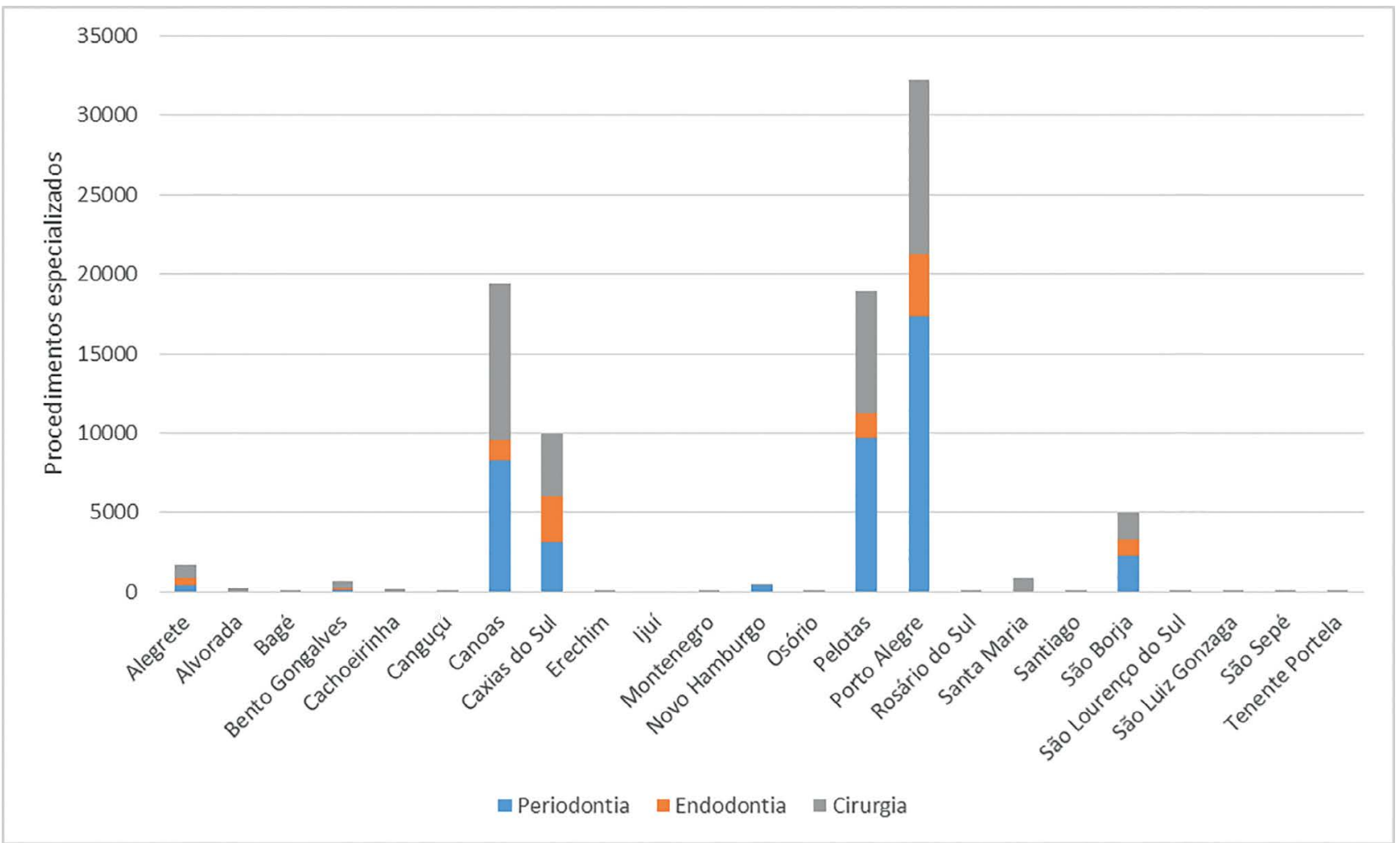

Figura 1 - Procedimentos especializados registrados no SIA SUS a partir de 2013 pelos municípios com CEOs cadastrados no CNES Fonte: SIA SUS. 


\section{Discussão}

Por meio desta pesquisa, foi possível verificar uma grande disparidade no registro dos procedimentos especializados entre os municípios estudados. Poucos municípios apresentaram registro da produção especializada compatível com a estabelecida, e uma parte dos municípios apresentou produção especializada nula ou insignificante.

Até o momento da pesquisa, nenhum estudo havia sido conduzido com a finalidade de investigar a produção odontológica especializada nos municípios com CEOs no RS. O entendimento de como a produção está distribuída no estado pode ser importante para o planejamento e a estruturação de políticas públicas de saúde, além de revelar a abrangência de novas políticas, como a Política Nacional de Saúde Bucal, o Brasil Sorridente. Tal programa, que se iniciou em 2004, faz parte da Saúde da Família e ampliou em $445 \%$ o número de equipes de saúde bucal em todo o Brasil, o que proporcionou uma expansão também da atenção secundária ${ }^{8}$.

Dessa forma, constatou-se que foram registrados mais de 90 mil procedimentos especializados pelo SUS nos municípios com CEOs durante os 28 meses observados. No entanto, observa-se que há uma grande variação no cumprimento das metas entre os municípios gaúchos. Algumas cidades (Pelotas, Caxias do Sul, Canoas) obtiveram índices que podem ser considerados muito bons, de outro modo, a maioria dos municípios (74\%) atingiu $50 \%$ ou menos das metas.

O presente estudo identificou uma grande concentração de CEOs em municípios de grande e médio porte populacional (Tabela 1), da mesma forma como é observado no restante do país, com um número maior nas regiões Nordeste e Sudeste do Bra$\mathrm{sil}^{15}$. A tendência de estabelecimento de CEOs em cidades de grande e médio porte pode dar-se porque elas naturalmente servem de referência aos municípios menores circundantes, seja em concentração de hospitais ou de profissionais da saúde, além de possibilitarem maior infraestrutura.

Dessa forma, notou-se uma elevada produção especializada nesses municípios (Tabela 2 e Figura 1). Porto Alegre foi a cidade que mais realizou procedimentos especializados, totalizando mais de 32 mil nos 28 meses analisados. Canoas e Pelotas produziram, aproximadamente, 19 mil procedimentos cada, no mesmo período de tempo. A grande produção de Porto Alegre pode ser justificada pelo fato de o município apresentar quatro CEOs tipo I e por se tratar da cidade mais populosa do estado, o que acarreta em maior demanda por serviços de saúde.

Celeste et al. ${ }^{16}$ (2014) analisaram municípios brasileiros com e sem CEOs, observando que variáveis socioeconômicas (PIB per capita e IDH) não influenciaram o desempenho dos CEOs. Por outro lado, municípios com indivíduos com melhores con- dições financeiras apresentaram maior número de procedimentos em todas as áreas. De certa forma, isso pode ser reflexo das desigualdades sociodemográficas de nossa sociedade, em que indivíduos mais ricos tendem a utilizar mais os serviços de saúde. Assim, por se tratar da capital, e da maior economia do estado, é esperado que um maior número de procedimentos seja realizado nos CEOs de Porto Alegre.

E importante observar que os municípios que apresentam pouca ou nenhuma produção em uma das áreas têm esse mesmo problema nos demais serviços, sendo a endodontia a área com maior déficit. Esse padrão de distribuição dos procedimentos pode ser observado também em distintas áreas. Periodontia e cirurgia obtiveram uma distribuição semelhante em todos os municípios que registraram tais procedimentos; da mesma maneira, os procedimentos de endodontia concentraram as menores produções em todos os municípios. Isto pode ser explicado, em parte, pelo maior tempo necessário para realização de tratamentos endodônticos, que necessitam muitas vezes de duas sessões clínicas, quando comparado com os demais procedimentos. Além disto, existe menor exigência de procedimento/mês na área de endodontia pela portaria normatizadora. Enquanto que para um CEO tipo I são necessários oitenta procedimentos/mês de cirurgia oral menor e sessenta de periodontia, somente 35 são necessários para endodontia. Entretanto, mesmo com meta quantitativa mais baixa, qualitativamente, a endodontia teve pior desempenho, com menor cumprimento de metas.

Apenas seis (26\%) dos 23 municípios atingiram mais do que $50 \%$ das metas cumpridas durante os 28 meses analisados. Pelotas obteve o melhor desempenho (91\%), seguido de Caxias do Sul $(90 \%)$ e Canoas (86\%). Porto Alegre, apesar de ter maior produção total dentre os municípios, ficou logo atrás, apresentando $66 \%$ das metas atingidas. Nessa avaliação, referente ao cumprimento das metas, os procedimentos básicos representaram $25 \%$ das metas. Quando esses procedimentos são coletados, eles se somam aos demais procedimentos do município, superestimando a produção. Essa é uma das desvantagens inerentes ao modelo utilizado neste estudo.

A única forma de se obter dados pelo SIA SUS é a partir da unidade amostral Município, e não é possível coletar dados das unidades básicas de saúde ou dos CEOs individualmente. Dessa forma, a produção básica das unidades básicas de saúde sobrepõe-se aos dados registrados nos CEOs, o que superestima nossa avaliação, principalmente nos procedimentos de atenção básica que devem ser ofertados a pessoas com necessidades especiais nos CEOs. A produção secundária, em geral, é produzida exclusivamente nos CEOs, porém algumas unidades básicas de saúde podem realizar procedimentos especializados, mesmo que isso não seja 
rotineiro, o que poderia superestimar de maneira mais discreta nossos dados. No entanto, mesmo com essa esperada superestimação, observou-se um registro expressivamente pobre ou nulo em alguns municípios, principalmente no que tange aos procedimentos especializados.

Como o registro no SIA SUS é ferramenta prevista em portaria interministerial e pactuada com o município no momento da habilitação do CEO, a medida deve ser cumprida. Tal registro é previsto como uma das principais ferramentas de monitoramento e controle por parte da gestão pública ${ }^{13}$. Dessa forma, os municípios devem manter o registro do sistema alimentado, correndo o risco de sanções caso isto não seja realizado.

Contudo, observou-se extensa deficiência no registro ou cumprimento das metas por grande número de municípios. Talvez isso se deva a problemas organizacionais de algumas cidades e de seus gestores, o que deve ser corrigido rapidamente. Políticas públicas devem ser planejadas locorregionalmente, o que demanda o envolvimento e comprometimento de diversas pessoas e órgãos ${ }^{17}$. Dessa forma, fatores organizacionais podem estar contribuindo para 0 baixo número de metas atingidas além das peculiaridades e dificuldades locais enfrentadas por diferentes municípios e gestores.

\section{Conclusão}

Com base na produção registrada no Data SUS, conclui-se que a maioria dos municípios gaúchos com CEO não atingiu as metas estabelecidas. Tendo em vista que municípios devem obrigatoriamente registrar sua produção no sistema, correndo o risco de sanções no financiamento caso não o façam, ressaltamos a importância de mais fiscalização pelos gestores. Além disso, destaca-se a impossibilidade de separar os dados registrados nas unidades básicas de saúde dos registrados nos CEOs, o que torna limitada a interpretação dos dados ao superestimá-los. A condução de análises com dados disponíveis pelo sistema de informações do SUS pode ser de extrema relevância ao fornecer um amplo banco de dados que pode ser utilizado como ferramenta por gestores e pesquisadores, contribuindo assim na estruturação de novas políticas públicas de saúde.

\section{Abstract}

Objectives: Dental Specialty Centers (CEOs) are facilities taken as reference for basic healthcare units, they should be linked to the National Register of Health Facilities (CNES), and offer specialized dental care to the public. Thus, this study aimed to describe the specialized dental production in the cities of the state of Rio Grande do Sul, Brazil, along with the CEOs, from the Outpatient Information System of the Unified Health System (SIA/SUS) data. Materials and methods: A retro- spective longitudinal study was carried out by analyzing secondary data from SIA/SUS. A survey was performed at the CNES of CEOs registered in cities of the state of Rio Grande do Sul. Dental production of these cities was then searched directly at the Department of Informatics of the Unified Health System (DATA/SUS) database and tabulated. From this research, a background series was created for each city. The minimum monthly production required varied according to the modalities of CEOs (Interministerial Decree 1464, of 2011), and the number of CEOs per city. Results: Periodontics procedures were most prevalent $(46.2 \%)$, followed by minor oral surgery $(41.3 \%)$, and endodontics $(12.5 \%)$. Regarding the fulfillment of goals, Pelotas was the city with the best performance $(91 \%)$, followed by Caxias do Sul (90\%), and Canoas (86\%). In contrast, 13 cities reached only $25 \%$ or less of the established goals. Conclusion: There is a major difference in either the fulfillment or recording of specialized dental procedures in the cities with CEOs in the state of Rio Grande do Sul.

Keywords: Public health. Health services assessment. Oral health services. Dental specialties. Oral health.

\section{Referências}

1. Brasil. Constituição (1988). Constituição da República Federativa do Brasil. Brasília: Senado; 1988.

2. Dias ACG. Caminhos para se pensar a promoção de saúde. Rev Latinoam Psicopatol Fundam 2013; 16(4):699-701.

3. Machado CV, Baptista TWF, Nogueira CO. Políticas de saúde no Brasil nos anos 2000: a agenda federal de prioridades. Cad Saude Publica 2011; 27(3):521-32.

4. Torres MF, Carvalho FR, Martins MD. Comparative study about the conceptions of health and disease between social science and dentistry students from a public university of Rio de Janeiro State. Cien Saude Colet 2011; 16(1):1409-15.

5. Paim J, Travassos C, Almeida C, Bahia L, Macinko J. The Brazilian health system: history, advances, and challenges. Lancet 2011; 377(9779):1778-97.

6. Brasil. Ministério da Saúde. Fundação Nacional de Saúde. Programa de Saúde da Família, Saúde dentro de Casa. Brasília (DF); 1994.

7. Scherer MDA, Marino SRA, Ramos FRS. Ruptures and resolutions in the health care model: reflections on the Family Health Strategy based on Kuhn's categories, Interface 2005; 9(16):53-66

8. Pucca Júnior GA, Gabriel M, Araujo ME, Almeida FC. Ten years of a National Oral Health Policy in Brazil: Innovation, Boldness, and Numerous Challenges. J Dent Res 2015; 94(10):1333-7.

9. Pucca Júnior GA. A política nacional de saúde bucal como demanda social. Ciên saúde colet 2006; 11(1):243-6.

10. Saliba NA, Nayme JGR, Moimaz SAS, Cecilio LPP, Garbin CAS. Organização da demanda de um Centro de Especialidades Odontológicas. Rev Odontol Unesp 2013; 42(1):317-23.

11. Figueiredo N, Goes PSA. Construção da atenção secundária em saúde bucal: um estudo sobre os Centros de Especialidades Odontológicas em Pernambuco, Brasil. Cad Saúde Pública 2009; 25(2):259-67.

12. Brasil. Ministério da Saúde. Portaria $\mathrm{n}^{\circ}$ 1.341, de 13 de junho de 2012. Define os valores dos incentivos de implantação e de custeio mensal dos Centros de Especialidades Odontológicas - CEO e dá outras providências. Brasília: Diário Oficial da União; 2012. 
13. Brasil. Ministério da Saúde. Portaria $\mathrm{n}^{\circ}$ 1.464, de 24 de junho de 2011. Altera o Anexo da Portaria no 600/GM/MS, de 23 de março de 2006, que institui o financiamento dos Centros de Especialidades Odontológicas (CEO). Brasília: Diário Oficial União; 2011.

14. Brasil. Ministério da Saúde. Secretaria de Atenção à Saúde. Departamento de Atenção Básica. Saúde Bucal. Brasília: Ministério da Saúde; 2008. 92 p. (Série A. Normas e Manuais Técnicos) (Cadernos de Atenção Básica, n. 17).

15. Goes PS, Figueiredo N, Neves JC, Silveira FM, Costa JF, Pucca Júnior GA et al. Vigilância à saúde bucal: a construção de um modelo integrado. Cad Saúde Pública 2012; 28 (Supl.):81-9.

16. Celeste RK, Moura FRR, Santos CP, Tovo MF. Análise da produção ambulatorial em municípios com e sem centros de especialidades odontológicas no Brasil em 2010. Cad Saúde Pública 2014; 30(3):511-21.

17. Paim JS, Teixeira CF. Política, planejamento e gestão em saúde: balanço do estado da arte. Rev Saúde Pública 2006; 40(Esp):73-8.

Endereço para correspondência:

Luiz Alexandre Chisini

Faculdade de Odontologia

Universidade Federal de Pelotas

Rua Gonçalves Chaves, 457, Sala 501

96015560 Pelotas, RS

Telefone: (53) 3222-4439

E-mail: luizalexandrechisini@hotmail.com alexandrechisini@gmail.com

Recebido: 23/09/15. Aceito: 05/01/16. 